\title{
Fatores reprodutivos associados ao excesso de peso em mulheres adultas atendidas pela Estratégia Saúde da Família
}

\author{
Reproductive factors associated with overweight in adult women \\ attended by the Family Health Strategy
}

Déborah Franco Gonçalves (https://orcid.org/0000-0002-9579-0628) ${ }^{1}$

Maria Teresa Bustamante Teixeira (https://orcid.org/0000-0003-0727-4170) ${ }^{1}$

Gulnar Azevedo Silva (https://orcid.org/0000-0001-8734-2799) ${ }^{2}$

Kristiane de Castro Dias Duque (https://orcid.org/0000-0001-7117-0945) ${ }^{1}$

Maria Lúcia Salim Miranda Machado (https://orcid.org/0000-0002-2786-8358) ${ }^{3}$

Luiz Cláudio Ribeiro (https://orcid.org/0000-0003-4409-2106) ${ }^{1}$
${ }^{1}$ Programa de PósGraduação em Saúde Coletiva, Universidade Federal de Juiz de Fora. R. José Lourenço Kelmer s/n, São Pedro. 36036-900 Juiz de Fora MG Brasil. deborahfranco25@ yahoo.com.br

${ }^{2}$ Programa de PósGraduação em Saúde Coletiva, Instituto de Medicina Social, Universidade do Estado do Rio de Janeiro. Rio de Janeiro RJ Brasil.

${ }^{3}$ Secretaria de Saúde, Prefeitura Municipal de Juiz de Fora. Juiz de Fora MG Brasil.

\begin{abstract}
Overweight stands out as a growing health problem in the population, resulting in individual and societal burdens. This study aimed to identify the association between reproductive factors and overweight in women of reproductive age attended by a Primary Health Care Unit (UAPS).This is a cross-sectional study with home capitation and data collection in two PHC Units, in the city of Juiz de Fora (MG), Brazil, in women aged 20 to 59 years, whose outcome was the overweight measured by the Body Mass Index. The prevalence of overweight was $61.0 \%$ among the 2,018 women included in the analysis. In the multivariate analysis, overweight was associated with the variables age at menarche before 12 years of age, having children, age greater than or equal to 30years, and hypertension. The prevalence of overweight in women who had menarche before 12 years of age was $12.4 \%$ higher than those who had menarche aged 12 years or more, and the prevalence of overweight in women who had children was $58.2 \%$ higher than those who never had any. There was a high prevalence of overweight in the adult female population, emphasizing the influence of reproductive factors.
\end{abstract}

Keywords Reproductive factors, Overweight, Women's health
Resumo $O$ excesso de peso se destaca como um problema de saúde crescente na população, resultando em ônus para o indivíduo e para a sociedade. O objetivo deste artigo é identificar a associação entre os fatores reprodutivos e o excesso de peso em mulheres de 20 a 59 anos de áreas cobertas pela Unidade de Atenção Primária à Saúde. Trata-se de um estudo transversal com captação domiciliar e coleta de dados em duas Unidades de Atenção Primária à Saúde, no município de Juiz de Fora, em mulheres de 20 a 59 anos, cujo desfecho foi o Excesso de Peso medido por meio do Indice de Massa Corporal. Entre as 2018 mulheres incluídas na análise, a prevalência de Excesso de Peso foi de 61,0\%. Na análise multivariada, $o$ Excesso de Peso apresentou associação com as variáveis idade da menarca antes dos 12 anos, ter filhos, idade maior ou igual a 30 anos e relato de hipertensão arterial. A prevalência de excesso de peso em mulheres que tiveram menarca antes dos 12 anos foi 12,4\% maior do que naquelas que a tiveram com 12 anos ou mais; enquanto que a prevalência de Excesso de Peso em mulheres que tiveram filhos foi 58,2\% maior do que entre as que nunca tiveram. Foi observada elevada prevalência de Excesso de Peso na população feminina adulta, com ênfase na influência dos fatores reprodutivos. Palavras-chave Fatores reprodutivos, Excesso de peso, Saúde da mulher 


\section{Introdução}

O excesso de peso é um grave problema de saúde pública e afeta diversos países no mundo, podendo ser resultado de hábitos alimentares inadequados, estilo de vida sedentário, fatores hormonais e fatores genéticos ${ }^{1,2}$. A maior preocupação acerca desse agravo é a sua relação com as doenças crônicas não transmissíveis como as doenças cardiovasculares e respiratórias crônicas, a diabetes e o câncer ${ }^{3}$.

De acordo com dados da Pesquisa de Orçamento Familiar - POF (2008-2009), com população em idade adulta, o excesso de peso foi diagnosticado em cerca de metade dos homens e das mulheres. O diagnóstico de obesidade foi encontrado em $12,5 \%$ dos homens e em $16,9 \%$ das mulheres, correspondendo a cerca de um quarto do total de casos de excesso de peso no sexo masculino e a um terço no sexo feminino 4 .

O Vigitel, sistema de monitoramento contínuo por inquérito telefônico, aponta uma tendência de aumento dos indicadores de excesso de peso em adultos nas capitais brasileiras. Entre os anos de 2006 e 2013 o estudo mostrou um aumento estatisticamente significante de $7,4 \%$ de sobrepeso e $47,1 \%$ de obesidade para ambos os sexos, faixas etárias, escolaridade e regiões ${ }^{5}$.

Dados de 2014 do Vigitel, que buscam descrever as prevalências dos fatores de risco e proteção para doenças crônicas, mostraram uma prevalência de $52,5 \%$ de excesso de peso na população adulta, $56,5 \%$ dos homens e $49,1 \%$ das mulheres. Entre estes, 17,9\% estavam obesos, com frequência semelhante entre os sexos 6 .

$\mathrm{O}$ excesso de peso é muito prevalente em mulheres adultas e pode interferir diretamente na qualidade de vida da população feminina. A história reprodutiva da mulher representada por fatores tais como idade da menarca, menopausa, gravidez, uso de contraceptivos e número de filhos podem resultar em um ganho de peso excessivo nessa fase da vida ${ }^{7,8}$.

Ainda que na literatura existam estudos sobre excesso de peso em mulheres adultas, não há consenso sobre quais fatores reprodutivos femininos se associam a este desfecho. Alguns autores apontam a associação positiva entre excesso de peso e fatores como menarca antes dos 12 anos, ter filhos e uso de contraceptivos ${ }^{7}$, entretanto, outros encontraram poucas associações a fatores reprodutivos, sendo a variável número de filhos a que mais apresentou associação com o excesso de peso $^{8-11}$.
O uso de contraceptivos hormonais, principalmente os injetáveis, apresentam associação com excesso de peso, como pode ser observado nos estudos de Lopez et al. ${ }^{12}$ e Dal'Ava et al..$^{13}$. Já outros estudos não apontam associação $0^{9,10}$, sendo necessários mais trabalhos que esclareçam a relação entre algumas variáveis relacionadas ao excesso de peso nessa população. A relação entre uso de contraceptivos e excesso de peso ainda não está bem definida na literatura ${ }^{7,9}$. As evidências sugerem que o aumento de peso pode estar associado ao uso dos contraceptivos hormonais ${ }^{13,14}$.

O objetivo do trabalho foi identificar a associação entre os fatores reprodutivos e o Excesso de Peso em mulheres em idade reprodutiva atendidas por Unidade de Atenção Primária à Saúde (UAPS) na cidade de Juiz de Fora, MG.

\section{Método}

Trata-se de um estudo epidemiológico de delineamento transversal com captação domiciliar e coleta de dados realizada em duas Unidades de Atenção Primária à Saúde, no município de Juiz de Fora, Minas Gerais. Este estudo integra a pesquisa "Avaliação de estratégias para o rastreamento do câncer do colo do útero em mulheres cobertas pela Estratégia de Saúde da Família no município de Juiz de Fora, Minas Gerais", aprovada pela Comissão de Ética do Instituto de Medicina Social (IMS) da Universidade do Estado do Rio de Janeiro (UERJ).

Para a participação na pesquisa foram elegíveis todas as mulheres da área de abrangência da UAPS na faixa etária de 20 a 59 anos, faixa etária alvo para o rastreamento citopatológico naquele momento, excluindo-se as grávidas, as virgens e as histerectomizadas. A partir de levantamento nas áreas de abrangência das UAPS, realizado pelos agentes comunitários de saúde (ACS), foram identificadas 4.516 mulheres nessa faixa etária. Todas foram convidadas a participar da pesquisa por meio de contato no domićlio feito pelos ACS.

Para as mulheres que não atenderam ao convite, foram realizadas outras duas tentativas de agendamento, por meio de nova visita domiciliar e/ou contato telefônico. Participaram do estudo 2.077 mulheres, o que correspondeu a $46 \%$ do universo. Na presente análise foram consideradas as 2.018 mulheres cujas medidas antropométricas foram aferidas.

A coleta de dados foi realizada por meio de um questionário que abordou fatores sociode- 
mográficos, apoio social, autoavaliação de estado de saúde, estilo de vida, morbidade, saúde da mulher e comportamento sexual, aplicado por profissionais de nível superior, previamente capacitados. Após a entrevista foram aferidas as medidas de pressão arterial e antropométricas e em seguida realizado o exame ginecológico com a coleta de material para o exame de Papanicolau, por profissionais médicos ou enfermeiros.

Para aferir as medidas de peso e altura foi utilizada balança eletrônica da marca "Tanita" com divisão de $0,1 \mathrm{~g}$, e a estatura por meio de um estadiômetro marca "Altura Exata" com divisão de $0,1 \mathrm{~cm}$. A balança esteve apoiada sobre superfície plana, firme e lisa, afastada da parede, travada e calibrada. Posicionavam-se no centro do equipamento, com o mínimo de roupa, descalça, ereta, com os pés juntos e os braços estendidos ao longo do corpo.

Para a avaliação do excesso de peso utilizouse o Índice de Massa Corporal (IMC), calculado dividindo o peso (medido em quilogramas) pela altura (medida em metros) ao quadrado. Para a análise, o IMC foi dividido em duas categorias: Sem Excesso de Peso (IMC $<25,0 \mathrm{Kg} / \mathrm{m}^{2}$ ) e Excesso de Peso $\left(\mathrm{IMC}>=25,0 \mathrm{Kg} / \mathrm{m}^{2}\right)^{15}$.

As variáveis independentes foram agrupadas segundo características socioeconômicas (situação conjugal, escolaridade, cor/raça, trabalho, salário mínimo per capita e auxílio Bolsa Família), de estilo de vida (consumo de álcool, prática de atividade física, assiste TV e tabagismo), presença de doenças crônicas não transmissíveis (hipertensão, diabetes, infarto ou AVC e depressão), fatores reprodutivos (idade da menarca, menopausa, uso de contraceptivos, contraceptivos hormonais, gravidez anterior e número de filhos) e faixa etária.

Foram realizadas analises bivariada com aplicação de teste Qui-quadrado e multivariada, com aplicação de modelos de regressão de Poisson, no programa Stata 11.0, tendo sido calculadas as Razões de Prevalência Bruta (RPB) e ajustadas (RPA) com respectivos Intervalos de Confiança de $95 \%$ (IC 95\%).

A análise multivariada foi realizada em duas etapas. A primeira etapa resultou em um modelo com análise prévia apenas com fatores reprodutivos, sendo consideradas significantes as variáveis que apresentaram valor de $\mathrm{p}<0,05$. Em seguida, as demais variáveis foram acrescentadas para verificar de que forma sua inclusão no modelo afetava a associação das variáveis reprodutivas com o Excesso de Peso.

\section{Resultados}

Nas 2.018 mulheres incluídas na análise, a média de idade foi de 38,42 anos (DP 11,04) e a média do IMC foi de 27,75 Kg/m² (DP 6,16; mínimo de $13,62 \mathrm{Kg} / \mathrm{m}^{2}$; máximo de $\left.83,36 \mathrm{Kg} / \mathrm{m}^{2}\right)$. Dentre as mulheres, $61 \%$ apresentaram Excesso de Peso, sendo que $30,0 \%$ apresentaram sobrepeso (IMC entre 25 e $\left.29,9 \mathrm{Kg} / \mathrm{m}^{2}\right)$ e $31,0 \%$ apresentaram obesidade (IMC $>=30 \mathrm{Kg} / \mathrm{m}^{2}$ ).

$\mathrm{Na}$ caracterização foram encontradas $61,0 \%$ de mulheres casadas, $55,6 \%$ de raça não branca, 54,3\% apresentavam ensino fundamental completo ou mais, $53,2 \%$ relataram trabalhar e $15,6 \%$ usufruíam do benefício do programa Bolsa Família. A mediana de rendimento mensal encontrada foi de R\$1.411,85, sendo que $41,8 \%$ de mulheres viviam com $1 / 2$ salário mínimo ou menos por mês.

A respeito dos fatores reprodutivos, mais da metade das mulheres (55,5\%) apresentavam menarca com 12 anos ou mais, 19,2\% estavam em menopausa, $87,3 \%$ da população já haviam engravidado e $85,6 \%$ tiveram filhos. A maior parte das mulheres relatou fazer uso de métodos contraceptivos $(68,5 \%)$, sendo que $66,4 \%$ disseram utilizar os do tipo hormonal.

Na Tabela 1 observa-se a distribuição das variáveis na análise bivariada, sendo que o Excesso de Peso teve relação com as seguintes: faixa etária maior do que 30 anos, ser casada, ter até ensino fundamental incompleto, raça não branca, trabalhar, não consumir álcool, não tabagismo, ter hipertensão, diabetes, infarto e depressão. Foi encontrada ainda elevada associação entre o Excesso de Peso e os fatores reprodutivos, sendo que a única variável que não se mostrou significante foi uso de contraceptivo.

A Tabela 2 apresenta os resultados da análise multivariada. A faixa etária apresentou associação com o Excesso de Peso, sendo que a prevalência em mulheres com 30 anos ou mais foi $22,1 \%$ maior do que em mulheres com menos idade e essa diferença é significativa.

As variáveis reprodutivas que mantiveram associação significativa com Excesso de Peso foram menarca antes dos 12 anos e ter filhos. A prevalência de Excesso de Peso em mulheres com idade da menarca maior ou igual a 12 anos foi $12,4 \%$ menor do que aquelas que a tiveram antes dessa idade. A prevalência de Excesso de Peso em mulheres que tiveram filhos foi $58,2 \%$ maior do que as que nunca tiveram.

Além das reprodutivas, outras variáveis que ainda mantiveram significância no modelo final 
Tabela 1. Número de casos, percentual de Excesso de Peso e valor de p por categoria, dado pelo teste Qui-quadrado para verificação da significância de associação com Excesso de Peso, segundo variáveis socioeconômicas e demográficas, de estilo de vida, doenças crônicas não transmissíveis e fatores reprodutivos femininos. Em Juiz de Fora, MG, Brasil.

\begin{tabular}{|c|c|c|c|}
\hline & \multicolumn{3}{|c|}{ Excesso de Peso $\left(\mathrm{IMC}>=25,0 \mathrm{Kg} / \mathrm{m}^{2}\right)$} \\
\hline & $\mathbf{N}$ & $\%$ & $\mathbf{p}$ \\
\hline Faixa Etária & & & $<0,001$ \\
\hline$<30$ anos & 532 & 46,6 & \\
\hline$\geq 30$ anos & 1486 & 62,2 & \\
\hline Situação Conjugal & & & $<0,001$ \\
\hline Casada & 1230 & 64,4 & \\
\hline Não Casada & 788 & 55,7 & \\
\hline Escolaridade & & & $<0,001$ \\
\hline Até Fundamental Incompleto & 912 & 66,1 & \\
\hline Fundamental Completo ou mais & 1084 & 56,6 & \\
\hline Cor / Raça & & & 0,014 \\
\hline Branca & 894 & 58,1 & \\
\hline Não Branca & 1119 & 63,4 & \\
\hline Trabalha & & & 0,005 \\
\hline Não & 943 & 64,3 & \\
\hline Sim & 1072 & 58,1 & \\
\hline Consumo de Álcool & & & 0,001 \\
\hline Não & 1151 & 64,1 & \\
\hline $\operatorname{Sim}$ & 865 & 56,9 & \\
\hline Tabagismo & & & $<0,001$ \\
\hline Não & 1578 & 63,6 & \\
\hline Sim & 425 & 51,1 & \\
\hline Hipertensão Arterial Sistêmica & & & $<0,001$ \\
\hline Não & 1521 & 54,8 & \\
\hline $\operatorname{Sim}$ & 485 & 81,0 & \\
\hline Diabetes & & & $<0,001$ \\
\hline Não & 1895 & 59,5 & \\
\hline $\operatorname{Sim}$ & 123 & 83,7 & \\
\hline Infarto ou AVC & & & 0,008 \\
\hline Não & 1963 & 60,5 & \\
\hline $\operatorname{Sim}$ & 55 & 72,2 & \\
\hline Depressão & & & 0,011 \\
\hline Não & 1545 & 59,5 & \\
\hline Sim & 473 & 66,0 & \\
\hline Idade da Menarca & & & $<0,001$ \\
\hline$<12$ anos & 885 & 65,8 & \\
\hline$\geq 12$ anos & 1103 & 56,8 & \\
\hline Menopausa & & & $<0,001$ \\
\hline Não & 1631 & 58,9 & \\
\hline $\operatorname{Sim}$ & 387 & 69,8 & \\
\hline Tipo de Contraceptivo & & & $<0,001$ \\
\hline Não Hormonal & 1339 & 65,1 & \\
\hline Hormonal & 679 & 52,9 & \\
\hline Já engravidou & & & $<0,001$ \\
\hline Não & 257 & 41,0 & \\
\hline Sim & 1761 & 63,9 & \\
\hline Teve Filhos & & & $<0,001$ \\
\hline Não & 290 & 41,7 & \\
\hline Sim & 1728 & 64,3 & \\
\hline
\end{tabular}


Tabela 2. Análise multivariada considerando o Excesso de Peso em mulheres adultas: Distribuição das razões de prevalência, intervalos de confiança e valor p segundo as variáveis significativas, em Juiz de Fora, MG, Brasil.

\begin{tabular}{|c|c|c|c|c|c|c|}
\hline Variável & RPBruta $^{*}$ & (IC - 95\%) & $\mathbf{p}$ & RPAjust $^{* *}$ & $(\mathrm{IC}-95 \%)$ & $\mathbf{P}$ \\
\hline \multicolumn{7}{|l|}{ Idade da Menarca } \\
\hline$<12$ anos & 1 & & & 1 & & \\
\hline$\geq 12$ anos & 0,946 & $0,921-0,972$ & $<0,001$ & 0,876 & $0,819-0,937$ & $<0,001$ \\
\hline \multicolumn{7}{|l|}{ Teve Filhos } \\
\hline Não & 1 & & & 1 & & \\
\hline Sim & 1,582 & $1,366-1,834$ & & 1,394 & $1,194-1,626$ & $<0,001$ \\
\hline \multicolumn{7}{|l|}{ Situação Conjugal } \\
\hline Não Casada & 1 & & & 1 & & \\
\hline Casada & 1,156 & $1,027-1,085$ & $<0,001$ & 1,108 & $1,030-1,193$ & 0,006 \\
\hline \multicolumn{7}{|l|}{ Raça } \\
\hline Branca & 1 & & & 1 & & \\
\hline Não Branca & 1,092 & $1,018-1,174$ & 0,015 & 1,076 & $1,005-1,153$ & 0,034 \\
\hline \multicolumn{7}{|c|}{ Hipertensão Arterial Sistêmica } \\
\hline Não & 1 & & & 1 & & \\
\hline Sim & 1,170 & $1,199-1,140$ & $<0,001$ & 1,326 & $1,240-1,418$ & $<0,001$ \\
\hline \multicolumn{7}{|l|}{ Diabetes Mellitus } \\
\hline Não & 1 & & & 1 & & \\
\hline Sim & 1,152 & $1,100-1,197$ & $<0,001$ & 1,147 & $1,048-1,256$ & 0,003 \\
\hline \multicolumn{7}{|l|}{ Faixa Etária } \\
\hline$<30$ anos & 1 & & & 1 & & \\
\hline$\geq 30$ anos & 1,133 & $1,097-1,170$ & $<0,001$ & 1,221 & $1,103-1,352$ & $<0,001$ \\
\hline
\end{tabular}

foram: casada, raça não branca, hipertensão, diabetes e 30 anos ou mais de idade. A prevalência de Excesso de Peso em mulheres casadas foi $10,8 \%$ maior do que nas não casadas e a prevalência em mulheres de raça não branca foi 9,2\% maior do que em brancas.

Em relação à presença de doenças crônicas, observou-se que a prevalência de Excesso de Peso em mulheres hipertensas foi 32,6\% maior do que em não hipertensas. A prevalência de Excesso de Peso em mulheres diabéticas foi $14,7 \%$ maior do que em não diabéticas.

As análises permitiram a comparação entre a Razão de Prevalência Bruta e a Razão de Prevalência Ajustada, para avaliar como as variáveis se comportaram na análise multivariada depois de realizada a análise isolada. Dessa forma, é possível dizer que a associação entre o Excesso de Peso e algumas variáveis ficou mais fraca, com a razão de prevalência ajustada mais próxima da unidade. Mesmo assim as variáveis continuaram significativas (Tabela 1).

\section{Discussão}

Conhecer mais sobre os fatores reprodutivos da mulher e sua influência no ganho Excessivo de Peso é crucial, uma vez que não há ainda um consenso nos estudos sobre o tema. No presente estudo 61\% das mulheres apresentaram Excesso de Peso, sendo 31\% obesidade, o que se torna motivo de preocupação, considerando a importância desse grupo populacional. As prevalências encontradas foram maiores do que aquelas mostradas em estudos transversais que também avaliaram fatores reprodutivos e Excesso de Peso em mulheres adultas de baixa renda ${ }^{7,8,10}$.

A proporção de Excesso de Peso entre aquelas que tinham menos de 30 anos foi $42 \%$ menor do que naquelas com mais de 30 anos de idade. Dados do Vigitel, que utiliza amostras probabilísticas da população adulta, também mostraram em 2014 um aumento das prevalências de excesso de peso e obesidade com o avanço da idade, principalmente entre 35 e 44 anos, chegando à prevalência de $58,6 \%$ de excesso de peso ${ }^{6}$. Outros estudos realizados com mulheres adultas encontraram elevadas prevalências de Excesso de Peso 
em mulheres em faixas etárias mais avançadas $s^{7-10}$, o que demonstra a necessidade de maior monitoramento dos indicadores com o aumento da idade, no intuito de prevenir agravos à saúde.

O Excesso de Peso teve relação com a situação conjugal da mulher, sendo maior a proporção entre as que possuíam companheiro. Estar solteira parece proteger a mulher dos distúrbios nutricionais, provavelmente devido a uma maior preocupação com a imagem corporal, a uma vida social mais ativa e a uma menor preocupação com os cuidados do lar e dos filhos, quando comparado às mulheres casadas ${ }^{7}$. Sá e Moura ${ }^{16} \mathrm{e}$ Andrade et al. ${ }^{17}$ também encontraram maior relação entre Excesso de Peso e ter companheiro, em estudos com população adulta.

A baixa escolaridade também apresentou associação com o Excesso de Peso, assim como em outros estudos com mulheres em situação socioeconômica semelhante ${ }^{10,18,19}$. Esse resultado demonstra que um limitado nível de compreensão pode afetar a adoção de estilos de vida mais saudáveis e a prevenção do ganho de peso.

A proporção de Excesso de Peso entre mulheres de raça não branca foi $9 \%$ maior do que as mulheres de raça branca na análise bivariada, mas perdeu significância na multivariada. Sá e Moura, também encontraram associação inversa entre Excesso de Peso e raça branca, ao utilizar dados de mulheres adultas que responderam ao inquérito do Vigitel em 2008. O resultado da análise bivariada para variável trabalha aponta para um resultado semelhante ao de Lins et al. ${ }^{8}$, que verificou que a atividade laboral leve pode ser protetora quanto ao Excesso de Peso. No entanto, quando controlado pelas demais variáveis, esse fator perdeu significância em ambos os estudos.

As mulheres que trabalhavam apresentaram menor proporção de Excesso de Peso sobre as que não trabalhavam, devido ao gasto energético maior durante a atividade. O mesmo foi encontrado em um trabalho realizado com mulheres em idade reprodutiva, que aponta que exercer atividade física leve pode ter efeito protetor sobre o Excesso de Peso ${ }^{8}$.

Um padrão alimentar adequado, caracterizado pelo consumo de frutas, hortaliças, carnes magras, lácteos desnatados, frutos secos e moderada ingestão de óleos vegetais e álcool, pode determinar um menor risco de doenças crônicas como a obesidade, a síndrome metabólica e as doenças cardiovasculares ${ }^{19,20}$. Em uma revisão sistemática, Farpour-Lambert et al. ${ }^{11}$ ressaltam que uma dieta balanceada deve ser recomendada no início da gravidez e mantida durante o pós-parto por ser eficaz na redução do ganho de peso gestacional e hipertensão gestacional em mulheres com obesidade.

No presente estudo não consumir álcool relacionou-se ao Excesso de Peso na análise bivariada, diferente do que foi encontrado por Sanvisens et al. ${ }^{21}$, que encontraram associação entre consumo de álcool e obesidade em mulheres. Em relação ao tabagismo, não fumar relacionou-se com excesso de peso, refutando alguns estudos que mostram que não há relação direta entre tabagismo e excesso de peso em mulheres ${ }^{10,18}$, devido à possível inibição do apetite causada pelo fumo ${ }^{22}$.

A avaliação de doenças crônicas merece atenção, uma vez que a proporção de Excesso de Peso em hipertensas foi $48 \%$ maior do que em não hipertensas, assim como encontrado por outros autores que encontraram relação direta entre hipertensão arterial e excesso de peso ${ }^{20}$. Em relação ao diabetes, a proporção de Excesso de Peso em mulheres com a doença foi $41 \%$ maior do que as que não tinham, semelhante ao estudo de Kearns et al. ${ }^{23}$, que encontrou associação entre diabetes e aumento do IMC.

A maioria das variáveis reprodutivas apresentou associação estatisticamente significativa com o Excesso de Peso na análise bivariada. A idade da menarca é o início da vida reprodutiva da mulher e nessa fase pode acontecer um acúmulo mais rápido de gordura corporal, levando ao ganho excessivo de peso. Como outros estudos ${ }^{7,24,25}$, foi encontrada associação entre menarca precoce e Excesso de Peso, sendo que mulheres com menarca antes dos 12 anos apresentaram proporção de Excesso de Peso 16\% maior em relação àquelas que a tiveram com 12 anos ou mais.

A menopausa também se mostrou associada ao Excesso de Peso, assim como encontrado por Rosa et al. ${ }^{9}$, cujo estudo mostrou que a proporção de sobrepeso e obesidade é maior em mulheres na menopausa, resultado esperado levando-se em consideração a mudança de composição corporal feminina nessa fase. Mulheres em menopausa tiveram proporção de Excesso de Peso 19\% maior do que aquelas que ainda não entraram na menopausa.

Frequentemente estudos mostram que ao utilizar métodos contraceptivos a mulher apresenta maior probabilidade de obesidade ${ }^{7,9}$, devido a componentes presentes nestes produtos ${ }^{26,27}$. Porém a proporção de Excesso de Peso em mulheres que usavam contraceptivos não hormonais foi $23 \%$ maior do que as que usavam contraceptivo hormonal, mas não se manteve significantemente associada na análise multivariada. 
Ficar grávida ou ter filhos também foram variáveis que apresentaram forte associação com o Excesso de Peso, aquelas que já engravidaram apresentaram uma proporção de Excesso de Peso $56 \%$ maior do que as que nunca estiveram grávidas e a proporção de Excesso de Peso em mulheres que já tiveram filhos foi $54 \%$ maior do que as que não tiveram filhos, associação também relatada no estudo de Ferreira e Benício ${ }^{10}$, com 11.961 mulheres brasileiras de 20 a 49 anos, em que o efeito da paridade sobre a obesidade foi significativo.

Após a análise multivariada com desfecho Excesso de Peso, algumas variáveis saíram do modelo de regressão por não permanecerem significantes. Isso ocorre porque durante a análise bivariada é considerada apenas a relação entre duas variáveis, já na multivariada a análise está sendo controlada pelas variáveis simultaneamente, de forma constante e numa mesma categoria de referência. Assim, ocorre uma mudança no comportamento da associação da variável com o desfecho.

As variáveis reprodutivas que tiveram destaque significativo no modelo final, considerando os demais fatores, foram idade da menarca me- nor do que 12 anos e ter filhos. A menarca precoce aumentou em 59\% o risco de obesidade, considerando o estudo de Correia et al. ${ }^{7}$ Outros autores demonstraram que a história de três ou mais gestações e menarca aos 11 anos ou menos tiveram uma prevalência $25 \%$ maior de obesidade abdominal ${ }^{24}$. A associação entre paridade e obesidade pode ser ainda do tipo dose-resposta, sendo mais pronunciada entre as mulheres que possuem filhos do que entre as nulíparas ${ }^{12,11}$.

Vale ressaltar, que por se tratar de um estudo transversal não foi possível inferir causalidade, tornando-se uma limitação, sendo difícil determinar a existência de uma sequência temporal entre exposição ao fator e o desenvolvimento da doença, pois acontece em um único momento. Os resultados encontrados devem ser interpretados com cautela, pois são voltados para esse grupo estudado.

Conclui-se que o Excesso de Peso é um problema com alta prevalência na população feminina adulta estudada e que os fatores reprodutivos associam-se ao seu desenvolvimento, sendo indicada uma adequada avaliação do diagnóstico nutricional, especialmente em mulheres que tiveram filhos e menarca precoce.

\section{Colaboradores}

DF Gonçalves: Concepção, análise estatística, metodologia e redação. MTB Teixeira: Pesquisa, concepção e revisão. GA Silva, KCD Duque e MLSM Machado: Pesquisa. LC Ribeiro: Análise estatística, metodologia, redação e revisão.

\section{Referências}

1. Popkin BM, Adair LS, Ng S. W. NOW AND THEN: The Global Nutrition Transition: The Pandemic of Obesity in Developing Countries. Nutrition Reviews 2012; 70(1):3-21.

2. Associação Brasileira para o Estudo da Obesidade e da Síndrome Metabólica (ABESO). Diretrizes brasileiras de obesidade 2009/2010. $3^{\text {a }}$ ed. Itapevi: AC Farmacêutica; 2009.

3. Organização Pan-Americana da Saúde (OPAS). Doenças crônico-degenerativas e obesidade: estratégia mundial sobre alimentação saudável, atividade física e saúde. Brasília: OPAS; 2003.

4. Instituto Brasileiro de Geografia e Estatística (IBGE). Pesquisa de orçamentos familiares - POF 2008-2009: Antropometria e estado nutricional de crianças, adolescentes e adultos no Brasil. Rio de Janeiro: IBGE; 2010.

5. Malta DC, Santos MAS, Andrade SSCA, Oliveira TP, Stopa SR, Oliveira MM, Jaime P. Tendência temporal dos indicadores de excesso de peso em adultos nas capitais brasileiras, 2006-2013. Cien Saude Colet 2016; 21(4):1061-1069. 
6. Malta DC, Stopa SR, Iser BPM, Bernal RTI, Claro RM, Nardi ACF, Reis AAC, Monteiro CA. Fatores de risco e proteção para doenças crônicas por inquérito telefônico nas capitais brasileiras, Vigitel 2014. Rev. bras. epidemiol 2015; 18(Supl. 2):238-255.

7. Correia LL, Silveira DMI, Silva AC, Campos JS, Machado MMT, Rocha HAL, Cunha AJLA, Lindsay AC. Prevalência e determinantes de obesidade e sobrepeso em mulheres em idade reprodutiva residentes na região semiárida do Brasil. Cien Saude Colet 2011; 16(1):133-145.

8. Lins APM, Sichieri R, Coutinho WF, Ramos EG, Peixoto MVM, Fonseca VM. Alimentação saudável, escolaridade e excesso de peso entre mulheres de baixa renda. Cien Saude Colet 2013; 18(2):357-366.

9. Rosa MI, Silva FML, Giroldi SB, Antunes GN, Wendland EM. Prevalência e fatores associados à obesidade em mulheres usuárias de serviços de pronto-atendimento do Sistema Único de Saúde no sul do Brasil. Cien Saude Colet 2011; 16(5):2559-2566.

10. Ferreira RAB, Benicio MHD. Obesidade em mulheres brasileiras: associação com paridade e nível socioeconômico. Revista Panam Salud Pública 2015; 37(4/5):337-342.

11. Farpour-Lambert NJ, Ells LJ, Martinez de Tejada B, Scott C. Obesity and weight gain in pregnancy and postpartum: an evidence review of lifestyle interventions to inform maternal and child health policies. Frontiers in Endocrinology 2018; 9:546.

12. Lopez LM, Edelman A, Chen M, Otterness C, Trussell J, Helmerhorst FM. Progestin-only contraceptives: effects on weight. Cochrane Database Syst Rev 2013; 7.

13. Dal'Ava N, Bahamondes L, Bahamondes MV, Bottura BF, Monteiro I. Body weight and body composition of depot medroxyprogesterone acetate users. Contraception 2014; 90(2):182-187.

14. Pantoja M, Medeiros T, Baccarin MC, Morais S, Fernandes AMS. Variation of weigth among users of the contraceptive with depot-medroxyprogesterone acetate according to body mass index in a six-year follow -up. Revista Brasileira de Ginecologia e Obstetrícia 2009; 31(8):380-384.

15. World Health Organization (WHO). Obesity: preventing and managing the global epidemic. Report of a World Health Organization Consultation. Geneva: World Health Organization. WHO Obesity Technical Report Series, no 284, 2000. [acessado 2016 Fev 23]. Disponível em: http://www.who.int/nutrition/publications/obesity/WHO_TRS_894/en/

16. Sá NNB, Moura EC. Excesso de peso: determinantes sociodemográficos e comportamentais em adultos, Brasil, 2008. Cad Saude Publica 2011; 27(7):13801392.

17. Andrade RG, Chaves OC, Costa DAS, Andrade ACS, Bispo S, Felicissimo MF, Friche AAL, Proietti FA, Xavier CC, Caiaffa WT. Excesso de peso em homens e mulheres residentes em área urbana: fatores individuais e contexto socioeconômico. Cad Saude Publica 2015; 31(Supl.):148-158.
18. Cristóvão MF, Sato APS, Fujimori E. Excesso de peso e obesidade abdominal em mulheres atendidas em Unidade da Estratégia Saúde da Família. Revista da Escola de Enfermagem da USP 2011; 45(n. esp. 2):1667-1672.

19. Lima NP, Horta BL, Motta JVS, Valença MS, Oliveira V, Santos TV, Gigante DP, Barros FC. Evolução do excesso de peso e obesidade até a idade adulta, Pelotas, Rio Grande do Sul, Brasil, 1982-2012. Cad Saude Publica 2015; 31(9):2017-2025.

20. Bressan J, Vidigal FC. Dieta na abordagem terapêutica da síndrome metabólica. Revista da Associação Brasileira de Nutrição - RASBRAN. 2014; 6(1):55-60.

21. Sanvisens A, Rivas I, Bolao F, Tor J, Rosón B, Rey-Joly C, Muga R. Gender and liver, nutritional and metabolic alterations of severe alcoholism: a study of 480 patients. Med Clin (Barc) 2011; 137(2):49-54.

22. Chatkin R, Chatkin JM. Tabagismo e variação ponderal: A fisiopatologia e genética podem explicar esta associação? Jornal Brasileiro de Pneumologia 2007; 33(6):712-719.

23. Kearns K, Dee A, Fitzgerald AP, Doherty E, Perry IJ. Chronic disease burden associated with overweight and obesity in Ireland: the effects of a small BMI reduction at population level. BMC Public Health 2014; 14(1):1-10.

24. Theodoro H, Rodrigues AD, Mendes KG, Liane RH, Paniz VMV, Olinto MTA. Reproductive characteristics and obesity in middle-aged women seen at an outpatient clinic in southern Brazil. Menopause 2012; 293(3):1022-1028.

25. Yang Y, Ma J, Fu L, Wang H, Dong B, Song Y, Shang XR, Liu FH, Zhang ZL. Association between early onset of menarche and anthropometry measurements among adolescent girls in China. Zhonghua Yu Fang Yi Xue Za Zhi 2013; 47(8):712-717.

26. Vasconcelos CMCS, Costa FS, Almeida PC, Araujo Júnior E, Sampaio HAC. Fatores de risco associados à retenção de peso seis meses após o parto. Revista Brasileira de Ginecologia e Obstetrícia 2014; 36(5):222227.

27. Negret A, Mercedes M, Despaigne L, Jesús M, Molina Hechavarría V, Sánchez Imbert N, Arias Carbonell MM. Efectos secundarios de los anticonceptivos hormonales en usuarias del método asistentes a las consultas de planificación familiar. MEDISAN 2013; 17(3):415-425.

Artigo apresentado em 01/03/2018

Aprovado em 12/11/2018

Versão final apresentada em 15/11/2018 